\title{
Functional expression and characterization in Xenopus laevis oocytes of the ABCG2 transporter derived from A549 human lung adenocarcinoma cells
}

\author{
DONG-GOO LEE ${ }^{1}$, HEE-JUNG CHO ${ }^{1}$, HEE YI ${ }^{1}$, SOO-MIN CHO ${ }^{1}, \mathrm{KYUL} \mathrm{JO}^{1}$, JIN-A PARK ${ }^{1}$, BYUNG-HWAN LEE ${ }^{2}$, \\ SUNG-HEE HWANG ${ }^{2}$, SANG MIN JEONG ${ }^{3}$, SEUNG-YEOL NAH ${ }^{2}$ and HO-CHUL SHIN ${ }^{1}$ \\ Departments of ${ }^{1}$ Veterinary Pharmacology and Toxicology, ${ }^{2}$ Physiology and ${ }^{3}$ Biochemistry and Molecular \\ Cell Biology, College of Veterinary Medicine, Konkuk University, Seoul 143-701, Republic of Korea
}

Received August 16, 2011; Accepted September 16, 2011

DOI: $10.3892 /$ or.2011.1490

\begin{abstract}
We cloned the ATP-binding cassette sub-family G member 2 (ABCG2) transporter, the most recently identified among several major human multidrug-resistance pumps, from A549 human lung adenocarcinoma cells in order to characterize its function and substrate specificity. In a previous report, we confirmed that a stem cell-like side population of A549 cells highly expressed the ABCG2 gene and had a unique ability to resist the anticancer drug methotrexate (MTX). In this study, ABCG2 cDNA was cloned by RT-PCR and converted into cRNA by an in vitro transcription system for expression in Xenopus laevis (X. laevis) oocytes. The transcribed cRNA of the ABCG2 gene was injected into the oocytes under the absence of cofactors or heterologous partner proteins or some lipids from the media. A high expression of ABCG2 was observed on the oocyte surface by immunofluorescence and confocal laser microscopy. We tested the functional effect of ABCG2 expression on drug efflux by directly injecting MTX into $X$. laevis oocytes. The drug concentration within the oocytes was quantified with LC-MS/MS; the analysis showed that the accumulation of MTX was significantly decreased in the $X$. laevis oocytes expressing ABCG2 compared with the control oocytes not expressing ABCG2. These findings show that the ABCG2 protein has an important role in the efflux of MTX through the cell membrane of $X$. laevis oocytes. Therefore, it might be that ABCG2, abundantly expressed in the stem cell population of A549 cells, can modulate resistance to MTX in lung cancer therapy.
\end{abstract}

Correspondence to: Dr Ho-Chul Shin, Department of Veterinary Pharmacology and Toxicology, College of Veterinary Medicine, Konkuk University, 1 Hwayang-dong, Gwangjin-gu, Seoul 143-701, Republic of Korea

E-mail: hshin@konkuk.ac.kr

Key words: ATP-binding cassette sub-family G member 2, A549 cells, methotrexate, cancer stem cell, Xenopus laevis oocyte

\section{Introduction}

Cells can become resistant to chemotherapy by overexpressing ATP-binding cassette (ABC) transporters, which use the energy from ATP hydrolysis to transport a wide variety of substrates across the cell membrane. ABC transporters have seven subfamilies, A to $\mathrm{G}$, which consist of several transmembrane proteins that use an energy source for the active extrusion of various drugs, endogenous materials, carcinogens and other xenobiotics across the cellular plasma membrane (1). Over the past 30 years, the functions of several ABC transporters were identified and include the absorption, distribution and excretion of drugs. These efflux transporters have a significant influence on drug pharmacokinetics and pharmacodynamics. Three human ABC transporters are most often associated with multidrug resistance phenomena in cancer cells: P-glycoprotein (P-gp)/MDR1/ABCB1, MRP1/ABCC1 and BCRP/MXR/ABCG2. P-gp (ABCB1) and MRP1 (ABCC1) (2-5), two of the most extensively studied ABC transporters, convey resistance to a wide variety of structurally unrelated cytotoxic agents $(6,7)$. However, several studies had difficulty confirming the MDR hypothesis in clinical trials (8). Despite the wealth of information collected about their biochemistry, the investigation of $\mathrm{ABC}$ transporter substrate specificity and efflux function in cancer cells has proven to be unexpectedly difficult (9).

The breast cancer resistance protein, BCRP/ABCG2, is the most recently identified half-molecule multidrug-resistance ATP-binding cassette transporter, however, it is not understood how this transporter affects drug resistance in a clinical setting (10). The human ABCG2 gene, containing 16 exons, 15 introns and spanning over $66 \mathrm{~kb}$, is located on chromosome 4q22 (11). The ABCG2 transporter is a 655-amino acid peptide with an ability to extrude a wide variety of biochemical compounds from cells (12). With high normal tissue expression in the brain endothelium, gastrointestinal tract, and placenta, ABCG2 is regarded as important in the protection from xenobiotics, in the regulation of oral bioavailability, and in the formation of part of the blood-brain barrier, the blood-testis barrier, and the maternal-fetal barrier. It can also be found in stem cells and some cancer cells (12). In multidrug resistant cancer 
cells, ABCG2 has been known to efflux potential anti-cancer drugs, such as methotrexate (MTX) and mitoxantrone (MX) $(11,13,14)$. In addition, ABCG2 has been linked to the stemness properties of cancer cells (15). A previous research study has shown that ABCG2-dependent drug efflux is associated with multidrug resistance in many cancers (16), with higher levels of DNA repair and lower levels of apoptosis (17).

In our previous studies $(18,19)$ we identified that ABCG2 expression was more than 2-fold higher in side population (SP) cells compared to non-side population cells, as determined by FACS with Hoechst 33342 staining. The ABCG2 transporter is known as a possible marker used to separate SP from non-SP cells, but it is not as well-studied compared with other ABC transporters (20). Therefore, we have cloned the ABCG2 cDNA from the A549 cell line and transformed it into the surface membrane of Xenopus laevis (X. laevis) oocytes to characterize the potential role of the ABCG2 efflux pump in multidrug resistance cancer cells.

\section{Materials and methods}

Materials. All chemicals and reagents were either molecular biology grade or immunohistochemistry grade. MTX was purchased from Sigma-Aldrich (St. Louis, MO, USA). The mouse monoclonal antibodies, BXP-21 and 34, were raised against the human BCRP and were produced by Abcam (Cambridge, England). Adult female $X$. laevis frogs were obtained from Xenopus 1, Inc. (Ann Arbor, MI, USA).

Preparation of human ABCG2 cDNA. The ABCG2 gene was isolated from A549 human lung adenocarcinoma cells by PCR with a single set of primers: 5'-TCC AGA TGG CTT CCA GTA AT-3' (forward) and 5'-GCA AGG GAA CAG AAA ACA AC-3' (reverse). The full-length ABCG2 cDNA was amplified with a one-step RT-PCR kit (Takara Bio, Shiga, Japan) and ligated into the pTARGET mammalian expression vector (Promega, Fitchburg, WI, USA). The amplified DNA was analyzed by gel electrophoresis and DNA sequencing. ABCG2 cDNA was also inserted by ligation (Toyobo, Osaka, Japan) into the pNBC 2.0 vector (Fig. 1A) for heterologous cRNA expression in a $X$. laevis oocytes system. All transformation and subcultivation procedures were performed with competent DH5- $\alpha$ E. coli cells (Real-Biotech Corp., Taipei, Taiwan). Plasmid DNA was prepared with the midi-prep kit (Qiagen, Hilden, Germany), and we checked the plasmid DNA with $1.0 \%$ agarose gel electrophoresis (Bio-Rad, Hercules, CA, USA) and DNA sequencing (Macrogen, Seoul, Korea).

cRNA microinjection and expression in X. laevis oocytes. The full-length ABCG2 cDNA was amplified with a one-step RT-PCR kit (Takara Bio) and it was utilized as an insert into the pTARGET mammalian expression vector. Plasmid DNA containing the desired ABCG2 cDNA insert was linearized by restriction enzyme $(S p h \mathrm{I})$ digestion and used as a template for synthesizing cRNA using the mMESSAGE mMACHINE kit (Ambion, Austin, TX, USA) and T7 RNA polymerase. Animal care and handling were carried out in accordance with the highest standards of institutional guidelines. X. laevis oocytes were prepared and maintained as previously described (27). Simultaneously, X. laevis oocytes were injected with $50 \mathrm{nl}$ of water per oocyte (hereafter referred to as Control oocytes) or $50 \mathrm{nl}$ of $1 \mu \mathrm{g} / \mu \mathrm{l} \mathrm{cRNA}$ solution. The oocytes were cultivated for 2-3 days after cRNA injection at $18^{\circ} \mathrm{C}$ in Barth's medium supplemented with $50 \mathrm{mg} / \mathrm{l}$ gentamicin (Sigma-Aldrich).

Western blot analysis. Groups of 24 oocytes were homogenized in $200 \mu \mathrm{l}$ of ice-cold lysis buffer (50 mM Tris- $\mathrm{HCl}, \mathrm{pH} \mathrm{8.0,}$ $150 \mathrm{mM} \mathrm{NaCl}, 1 \% \mathrm{NP}-40$ and $0.25 \%$ sodium deoxycholate) per group in the presence of a protease inhibitor cocktail (SigmaAldrich) and were then sonicated for $5 \mathrm{sec}$ at low power and vortexed. The homogenate was centrifuged at $1,000 \mathrm{x}$ g at $4^{\circ} \mathrm{C}$ for $10 \mathrm{~min}$, and the supernatant was centrifuged again. The protein concentration of the supernatant was determined by the BCA method. A volume of supernatant containing $25 \mu \mathrm{g}$ of extracted protein was subjected to $10 \%$ SDS-polyacrylamide gel electrophoresis and blotted by electrotransfer onto a polyvinylidene difluoride membrane. The blots were blocked with $5 \%$ non-fat dry milk and probed at $4{ }^{\circ} \mathrm{C}$ overnight with a 1:1,000 dilution of the BXP-21 monoclonal antibody to BCRP (Abcam). The blots were then incubated at room temperature for $1 \mathrm{~h}$ with a horseradish peroxidase-conjugated sheep antimouse IgG secondary antibody (Abcam) and were imaged with enhanced chemiluminescent detection (Amersham Biosciences, Uppsala, Sweden) using the Las-3000 (Fujifilm, Tokyo, Japan) analyzer and the Multi-Gauge software.

Immunofluorescence and confocal laser microscopy. Oocytes injected with water or ABCG2 cRNA were incubated for 3 days, fixed in phosphate-buffered saline (PBS, $\mathrm{pH}$ 7.4) with $10 \%$ formaldehyde and immersed overnight in PBS with $30 \%$ sucrose. Groups of 3-4 oocytes were frozen in OCT compound (Tissue-Tek; Sakura Finetek Europe, Zoeterwoude, The Netherlands), and $25 \mu \mathrm{m}$ sections were collected on gelatincoated slideglasses. Sections on slideglasses were inverted onto $50 \mu \mathrm{l}$ of primary antibody, BXP-34 (Abcam), diluted 1:50 in buffer A (PBS with 1\% bovine serum albumin) for $1 \mathrm{~h}$ at room temperature in a moisture chamber and were rinsed three times with buffer A for $10 \mathrm{~min}$. The sections were probed with fluorescein isothiocyanate-conjugated goat anti-mouse IgG (Abcam) diluted 1:100 in buffer A and were rinsed three times with buffer A for $10 \mathrm{~min}$ (21). Sections were mounted in aqueous anti-fade mounting medium (Biomeda Co., Foster City, CA, USA), and immunofluorescence was observed with a confocal scanning laser microscope (Olympus FV-1000, Tokyo, Japan) at x100 magnification. Using an initial section from an oocyte injected with ABCG2 cRNA, the background fluorescence was set to zero, and the brightest fluorescence was set to saturation. These parameter settings were used for all subsequent sections, and the autolevel was adjusted by Adobe Photoshop 7.0.

Determination of MTX efflux in X. laevis oocytes. ABCG2 cRNA-injected and water-injected $X$. laevis oocytes were cultivated in uptake buffer at room temperature for 3 days and then, $50 \mathrm{nl}$ of $25 \mu \mathrm{M}$ MTX was injected directly into each oocyte. Thereafter, 12 oocytes from each treatment group were transferred to $100 \mu \mathrm{l}$ of uptake buffer and incubated for $2 \mathrm{~h}$. After incubation, the oocytes were washed four times with ice-cold uptake buffer. Twelve oocytes from each group were transferred and homogenized in $100 \mu \mathrm{l}$ of mobile phase 


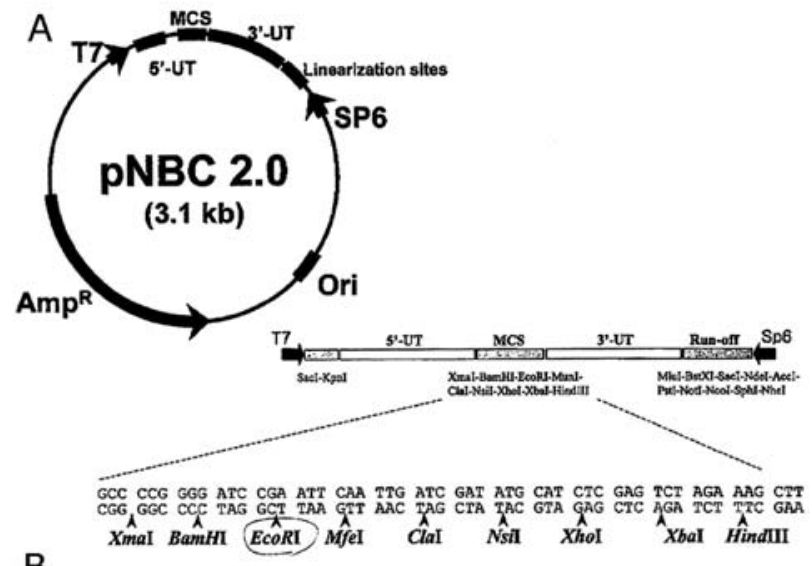

B

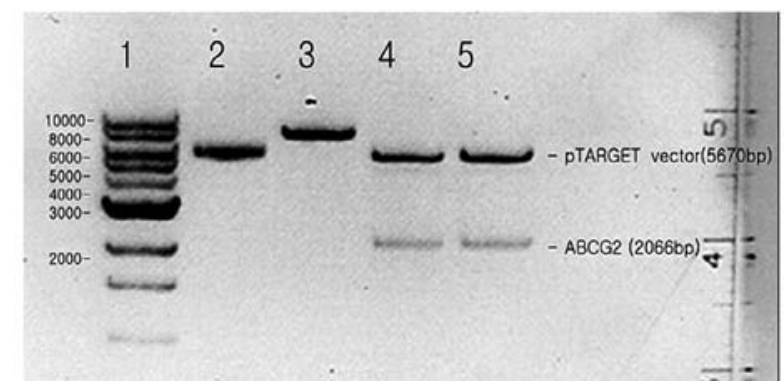

Figure 1. Plasmid construction of the full-length cDNA. (A) pNBC 2.0 $X$. laevis oocyte expression vector. (B) Lane 1, 1 kb DNA ladder (Takara Bio, Shiga, Japan); lane 2, pTARGET plasmid harboring ABCG2; lane 3, BamHI digest of lane 2; lane 4, BamHI-EcoRI double digest of lane 2; and lane 5, EcoRI digest of lane 2 .

solution, a mixture with an acetonitrile: ammonium formate ratio of 18:82 containing $0.1 \%$ formic acid, and the homogenate was centrifuged at $4^{\circ} \mathrm{C}$ for $20 \mathrm{~min}$ at $10,000 \mathrm{rpm}$. The resulting supernatant $(100 \mu \mathrm{l})$ and $100 \mu \mathrm{l}$ of uptake buffer were combined, filtered and subjected to LC-MS/MS (liquid chromatography with tandem mass spectrometry) analysis.

Detection and quantification of MTX by LC-MS/MS. The LC-MS/MS system was composed of an Agilent 1100 HPLC system (Agilent Technologies, Waldbronn, Germany) and an API2000 MS/MS system (Applied Biosystems/MDS SCIEX, Concord, Ontario, Canada) using electrospray ionization in positive ion scan mode. Chromatographic separation was achieved by using a mobile phase of acetonitrile: $1 \mathrm{mM}$ ammonium formate containing $0.1 \%$ formic acid (18:82, $\mathrm{v} / \mathrm{v})(22)$ delivered at a flow rate of $0.2 \mathrm{ml} / \mathrm{min}$ through an analytical column (Waters, XTerra MS $\mathrm{C}_{18}, 5 \mu \mathrm{m}$ particle size, $2.1 \times 150 \mathrm{~mm})$. The column temperature was maintained at $30^{\circ} \mathrm{C}$. The column effluent was monitored at the following transitions: MTX m/z 455.4 $\rightarrow 308.0$ with a dwell time of $150 \mathrm{~ms}$. The spray gas was nitrogen set at a pressure of $100 \mathrm{psi}$. The curtain gas and collision gas were both nitrogen and were both set at a pressure of 50 psi. The ion spray voltage was set at $5,500 \mathrm{~V}$ and the temperature was set to $350^{\circ} \mathrm{C}$.

\section{Results}

ABCG2 cDNA confirmation. Full-length ABCG2 cDNA (2,066 bp, which was modified to insert of Kozack sequence for

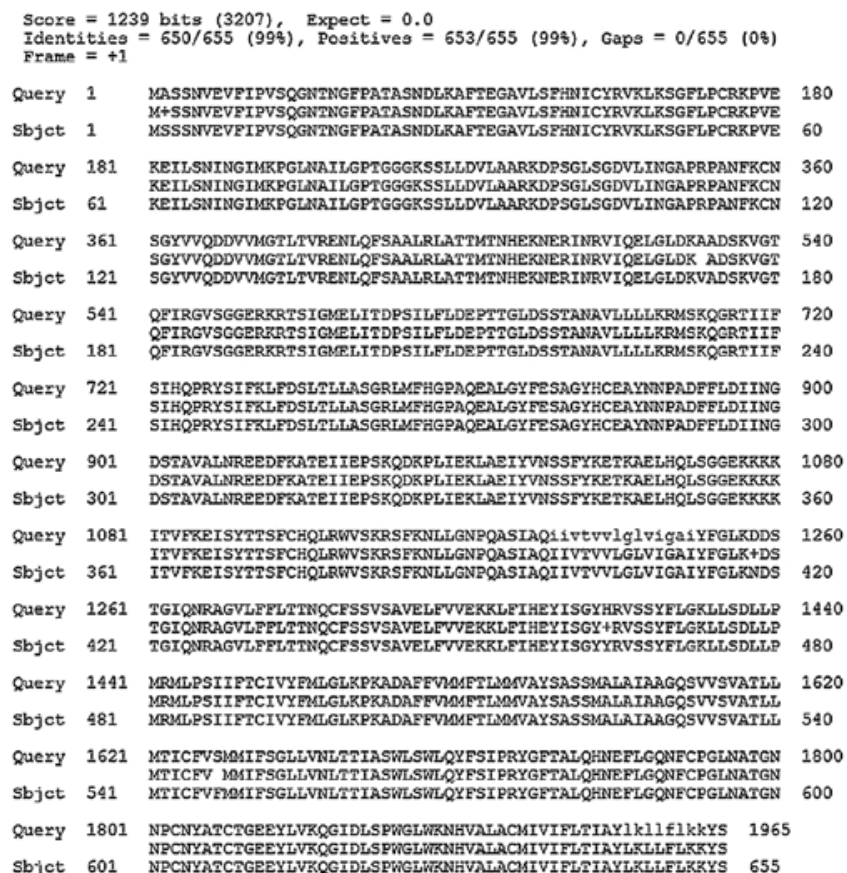

Figure 2. BLASTx analysis of the ABCG2-pTARGET plasmid. The confirmed nucleotide sequence of $\mathrm{ABCG} 2$ was translated into its amino acid sequence (upper line). The amino acid sequence exactly matched the human ABCG2 amino acid sequence (accession; NM_004827).

high transfection efficacy as described in Materials and methods) was generated by RT-PCR and inserted into the pTARGET vector $(5,670 \mathrm{bp})$. We utilized full-length ABCG2 cDNA for the competent DH5- $\alpha$ E. coli cell transformation. We confirmed the plasmid of ABCG2 using restriction enzymes (BamHI, EcoRI) digestion, gel electrophoresis and UV scanning (Fig. 1B) as well as DNA sequencing. The sequencing result matched the wild-type sequence of ABCG2 (Fig. 2). The ABCG2 insert was separated from the pTARGET vector, inserted into the $\mathrm{pNBC}$ 2.0 vector and transformed in $X$. laevis oocytes.

cRNA synthesis and in vitro transcription. The pNBC 2.0 vector containing the ABCG2 insert was linearized by $S p h \mathrm{I}$ restriction enzyme digestion. The linearized plasmid was transcribed to cRNA by an in vitro transcription kit. The amount of cRNA was confirmed by gel electrophoresis.

ABCG2 expressed in X. laevis oocytes localizes to the surface membrane. In vitro transcribed cRNA was quantified prior to injection into $X$. laevis oocytes. One experimental group of 30 oocytes received an injection of water (control oocytes). The other experimental group of 30 oocytes received an injection of cRNA (ABCG2+pNBC oocytes). Four oocytes from each group were fixed and made into $25 \mu \mathrm{m}$ cryosections as described in Materials and methods. Exposure of these sections to the BXP-34 monoclonal antibody, directed against ABCG2, resulted in immunofluorescence only in the cell membrane of oocytes expressing ABCG2 as detected by confocal fluorescence microscopy (Fig. 3A). Control oocytes showed no immunofluorescence. Western blot analysis of oocytes injected with ABCG2 cRNA at the time of the immunofluorescence 

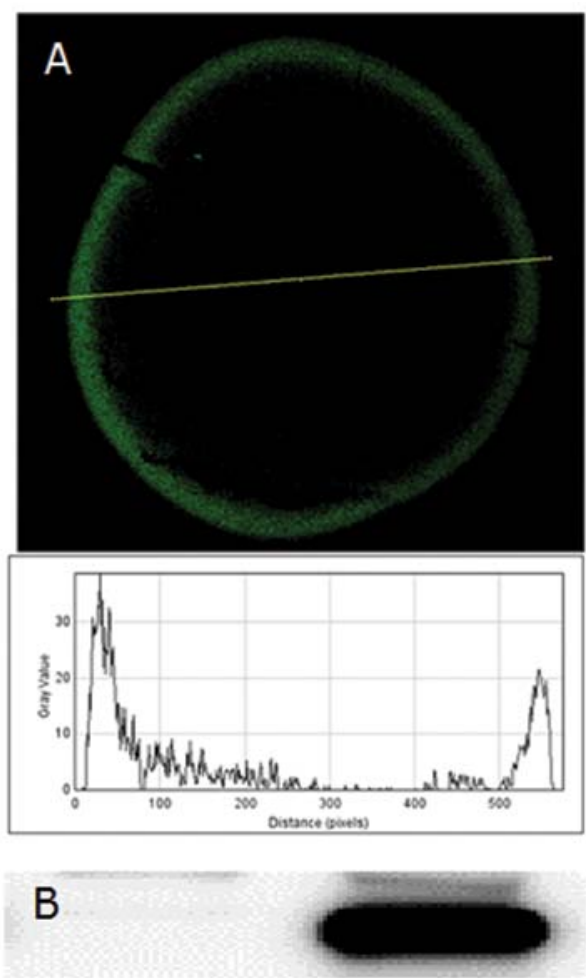

Figure 3. Confocal immunofluorescence microscopy of $X$. laevis oocyte injected with $50 \mathrm{nl}$ of cRNA of ABCG2. (A) Distribution of ABCG2 at the oocyte surface. (B) Cell lysate $(25 \mu \mathrm{g})$ from the oocytes injected with ABCG2 cRNA. After electrophoresis and blotting, the blots were probed with the BXP-21 antibody.

experiments confirmed robust expression of $\mathrm{ABCG} 2$ protein with a molecular mass of $70 \mathrm{kDa}$ (Fig. 3B).

Accumulation andefflux of MTX inoocytes expressing ABCG2. To determine whether functional ABCG2 was expressed in $X$. laevis oocytes, we monitored the accumulation of MTX in control and in ABCG2-injected oocytes. Oocytes expressing ABCG2 showed a significant reduction in the accumulation of MTX over a $2 \mathrm{~h}$ period. After background subtraction, MTX accumulation in the ABCG2-expressing oocytes was ca. 80\% of the control group as measured by LC-MS/MS (Fig. 4).

\section{Discussion}

This research extends previous functional protein analyses in SP cells in order to confirm the relationship between the up-regulation of specific genes and multidrug resistance. When conducting a functional study of upregulated genes, we performed our experiment with ABCG2 first because it was the most interesting gene of SP cells in our previous study. Though known as a potential marker of SP cells (20), we focused on ABCG2 as a cell-surface, ABC-protein family transporter that may mediate the efflux of anticancer agents, thereby reducing intracellular drug concentrations.

The ABCG2 gene spans over $66 \mathrm{~kb}$ and is made up of 16 exons and 15 introns; the resulting protein is 655 amino acids long and runs as a $72 \mathrm{kDa}$ protein on an SDS-PAGE gel under reducing conditions (23). Fluorescence in situ hybridization studies with a bacterial artificial chromosome probe containing

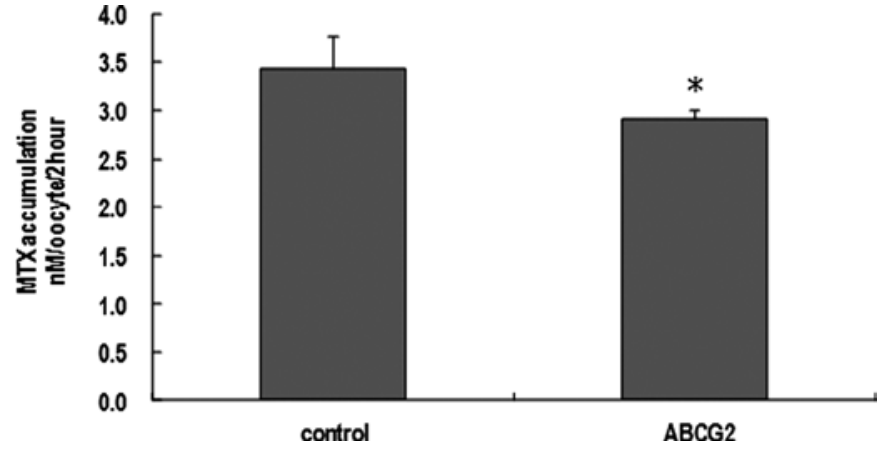

Figure 4. Uptake of MTX in control X. laevis oocytes and in ABCG2expressing oocytes. MTX, $50 \mathrm{nl}$ of $25 \mu \mathrm{M}$, was directly injected into each oocyte $\left(\mathrm{n}=12\right.$ oocytes, $\left.{ }^{*} \mathrm{P}<0.05\right)$.

ABCG2 localized the gene to chromosome $4 \mathrm{q} 21-4 \mathrm{q} 22$ in a cell with a normal chromosome 4 (24). In the past 10 years, many research articles and reviews have described experiments on the ABCG2/BCRP/MXR transporter. For example, physiological and pharmacological roles of ABCG2 were revealed by the study of ABCG2 knockout mice, including the study of their blood-brain, blood-testis and blood-fetal barriers (25). These functions indicate a primary biological role for ABCG2 in the protection of these cells from xenobiotics. However, several other physiological functions have been observed, including the extrusion of porphyrins and/or porphyrin conjugates from hematopoietic cells, liver and harderian glands (26) as well as the secretion of vitamin $B_{2}$ and other vitamins into breast milk (27). Various compounds have been tested as inhibitors of the ABCG2 transporter, and estrogenic compounds, including estrone, several tamoxifen derivatives, phytoestrogens and flavonoids, have been shown to reverse ABCG2-mediated drug resistance (28). Functional SNPs and inhibitory agents of BCRP modulate the in vivo pharmacokinetics and pharmacodynamics of BCRP substrates (29). Screening methods and 3D-QSAR (quantitative structure-activity relationship) allow the design of ABCG2 inhibitors and may provide promising drug candidates for clinical trials (30). However, the physiological significance of these processes and functional studies have been difficult to establish, indicating that there is still a lot to learn about this intriguing protein, ABCG2.

We cloned the ABCG2 gene, confirmed its sequence and transformed it using the mammalian expression vector pTARGET. We anticipated some point mutations within the ABCG2 gene because it has a relatively large portion of SP cells compared to other cell lines, but the sequence of ABCG2 in the A549 cells was the same as its wild-type sequence. After the transfection of ABCG2 into X. laevis oocytes, using immunofluorescence and confocal laser microscopy, we confirmed that the location of the ABCG2 protein was in the plasma membrane. The location of the ABCG2 protein was similar to that found previously (21). Furthermore, the efflux function of ABCG2 $(31,32)$ was also confirmed by testing the accumulation of the substrate MTX. Therefore, we conclude that ABCG2 may modulate resistance to MTX in lung cancer therapy. We believe that the X. laevis oocyte expression system is an effective means of studying ABCG2 function and substrate specificity. 


\section{Acknowledgements}

This study was supported by the Korea Research Foundation Grant (KRF-2007-331-E00251).

\section{References}

1. Dean M, Rzhetsky A and Allikmets R: The human ATP-binding cassette (ABC) transporter superfamily. Genome Res 11: 1156-1166, 2001.

2. Gottesman MM, Fojo T and Bates SE: Multidrug resistance in cancer: role of ATP-dependent transporters. Nat Rev Cancer 2: $48-58,2002$.

3. Chen CJ, Chin JE, Ueda K, et al: Internal duplication and homology with bacterial transport proteins in the mdrl (P-glycoprotein) gene from multidrug-resistant human cells Cell 47: 381-389, 1986.

4. Cole SP, Bhardwaj G, Gerlach JH, et al: Overexpression of a transporter gene in a multidrug-resistant human lung cancer cell line. Science 258: 1650-1654, 1992.

5. Kruh GD, Zeng H, Rea PA, et al: MRP subfamily transporters and resistance to anticancer agents. J Bioenerg Biomembr 33 493-501, 2001.

6. Borst P, Evers R, Kool M and Wijnholds J: The multidrug resistance protein family. Biochim Biophys Acta 1461: 347-357, 1999.

7. Ling V: Multidrug resistance: molecular mechanisms and clinical relevance. Cancer Chemother Pharmacol 40 (Suppl) S3-S8, 1997.

8. Leonard GD, Fojo T and Bates SE: The role of ABC transporters in clinical practice. Oncologist 8: 411-424, 2003.

9. Szakacs G, Paterson JK, Ludwig JA, Booth-Genthe C and Gottesman MM: Targeting multidrug resistance in cancer. Nat Rev Drug Discov 5: 219-234, 2006.

10. Doyle LA, Yang W, Abruzzo LV, et al: A multidrug resistance transporter from human MCF-7 breast cancer cells. Proc Natl Acad Sci USA 95: 15665-15670, 1998.

11. Allikmets R, Schriml LM, Hutchinson A, Romano-Spica V and Dean M: A human placenta-specific ATP-binding cassette gene $(\mathrm{ABCP})$ on chromosome $4 \mathrm{q} 22$ that is involved in multidrug resistance. Cancer Res 58: 5337-5339, 1998.

12. Staud F and Pavek P: Breast cancer resistance protein (BCRP/ ABCG2). Int J Biochem Cell Biol 37: 720-725, 2005.

13. Brangi M, Litman T, Ciotti M, et al: Camptothecin resistance: role of the ATP-binding cassette (ABC), mitoxantrone-resistance half-transporter (MXR), and potential for glucuronidation in MXR-expressing cells. Cancer Res 59: 5938-5946, 1999.

14. Miyake K, Mickley L, Litman T, et al: Molecular cloning of cDNAs which are highly overexpressed in mitoxantrone-resistant cells: demonstration of homology to ABC transport genes. Cancer Res 59: 8-13, 1999.

15. Krishnamurthy P, Ross DD, Nakanishi T, et al: The stem cell marker Bcrp/ABCG2 enhances hypoxic cell survival through interactions with heme. J Biol Chem 279: 24218-24225, 2004.

16. Lou H and Dean M: Targeted therapy for cancer stem cells: the patched pathway and ABC transporters. Oncogene 26: 1357-1360, 2007.
17. Zhou S, Schuetz JD, Bunting KD, et al: The ABC transporter $\mathrm{Bcrp} 1 / \mathrm{ABCG} 2$ is expressed in a wide variety of stem cells and is a molecular determinant of the side-population phenotype. Nat Med 7: 1028-1034, 2001.

18. Seo DC, Sung JM, Cho HJ, et al: Gene expression profiling of cancer stem cell in human lung adenocarcinoma A549 cells. Mol Cancer 6: 75, 2007.

19. Sung JM, Cho HJ, Yi H, et al: Characterization of a stem cell population in lung cancer A549 cells. Biochem Biophys Res Commun 371: 163-167, 2008

20. Bunting KD: ABC transporters as phenotypic markers and functional regulators of stem cells. Stem Cells 20: 11-20, 2002.

21. Nakanishi T, Doyle LA, Hassel B, et al: Functional characterization of human breast cancer resistance protein (BCRP, ABCG2) expressed in the oocytes of Xenopus laevis. Mol Pharmacol 64: 1452-1462, 2003.

22. Guo P, Wang X, Liu L, Belinsky MG, Kruh GD and Gallo JM: Determination of methotrexate and its major metabolite 7-hydroxymethotrexate in mouse plasma and brain tissue by liquid chromatography-tandem mass spectrometry. J Pharm Biomed Anal 43: 1789-1795, 2007.

23. Bailey-Dell KJ, Hassel B, Doyle LA and Ross DD: Promoter characterization and genomic organization of the human breast cancer resistance protein (ATP-binding cassette transporter G2) gene. Biochim Biophys Acta 1520: 234-241, 2001.

24. Knutsen T, Rao VK, Ried T, et al: Amplification of 4q21-q22 and the MXR gene in independently derived mitoxantrone-resistant cell lines. Genes Chromosomes Cancer 27: 110-116, 2000.

25. Vlaming ML, Lagas JS and Schinkel AH: Physiological and pharmacological roles of ABCG2 (BCRP): recent findings in Abcg2 knockout mice. Adv Drug Deliv Rev 61: 14-25, 2009.

26. Jonker JW, Musters S, Vlaming ML, et al: Breast cancer resistance protein (Bcrp1/Abcg2) is expressed in the harderian gland and mediates transport of conjugated protoporphyrin IX. Am J Physiol Cell Physiol 292: C2204-C2212, 2007.

27. van Herwaarden AE, Wagenaar E, Merino G, et al: Multidrug transporter $\mathrm{ABCG} 2 /$ breast cancer resistance protein secretes riboflavin (vitamin B2) into milk. Mol Cell Biol 27: 1247-1253, 2007.

28. Noguchi K, Katayama K, Mitsuhashi J and Sugimoto Y: Functions of the breast cancer resistance protein (BCRP/ABCG2) in chemotherapy. Adv Drug Deliv Rev 61: 26-33, 2009.

29. Kondo C, Suzuki H, Itoda M, et al: Functional analysis of SNPs variants of BCRP/ABCG2. Pharm Res 21: 1895-1903, 2004

30. Nicolle E, Boumendjel A, Macalou S, et al: QSAR analysis and molecular modeling of ABCG2-specific inhibitors. Adv Drug Deliv Rev 61: 34-46, 2009.

31. Honjo Y, Hrycyna CA, Yan QW, et al: Acquired mutations in the MXR/BCRP/ABCP gene alter substrate specificity in MXR/ BCRP/ABCP-overexpressing cells. Cancer Res 61: 6635-6639, 2001.

32. Saito H, Hirano H, Nakagawa H, et al: A new strategy of highspeed screening and quantitative structure-activity relationship analysis to evaluate human ATP-binding cassette transporter ABCG2-drug interactions. J Pharmacol Exp Ther 317: 1114-1124, 2006. 